\title{
Editorial: feasibility, complications, and cosmetic outcomes of immediate autologous fat grafting during breast-conserving surgery for early-stage breast cancer
}

\author{
Alireza Hamidian Jahromi^^, Sydney R. Horen^ \\ Division of Plastic and Reconstructive Surgery, Rush University Medical Center, Chicago, IL, USA \\ Correspondence to: Alireza Hamidian Jahromi, MD, MRCS. Clinical Instructor, Plastic and Reconstructive Surgery Department, Rush University \\ Medical Center, Chicago, IL, USA. Email: alirezahamidian@yahoo.com. \\ Comment on: Li H, Wang Y, Yang D, et al. Clinical study of the feasibility, complications, and cosmetic outcomes of immediate autologous fat grafting \\ during breast-conserving surgery for early-stage breast cancer in China. Gland Surg 2021;10:2386-97.
}

Submitted Aug 19, 2021. Accepted for publication Sep 07, 2021.

doi: $10.21037 /$ gs-21-563

View this article at: https://dx.doi.org/10.21037/gs-21-563

Autologous fat grafting (AFG) is a technique that has gained significant popularity over the recent years mostly to help achieve a more natural looking and youthful body/face contour and correct the deformities caused by oncologic resection, surgical contouring, and trauma (1). Since its introduction in the 1890's, AFG has undergone multiple refinements to maximize safety and efficacy. Currently, the uses of AFG include, but are not limited to, facial rejuvenation (i.e., in aging patients to replace subcutaneous tissue loss), craniofacial syndromes resulting in soft tissue defects in pediatric patients, gluteal augmentation (i.e., "Brazilian Butt Lift"), breast enhancement (i.e., in cosmetic breast augmentation with implant placement or free tissue transfer), and oncologic breast reconstruction following breast conserving surgery (BCS) or mastectomy (2-5).

The use of AFG in the setting of breast reconstruction following BCS has long been a topic of debate. In 1983, Illouz published a report on liposuction techniques which allowed for the harvest of fat for AFG to the breasts (6). However, soon after, the American Society of Plastic and Reconstructive Surgeons (ASPS) denounced AFG to the breast in the setting of BCS, stating that it may lead to an increased risk of cancer and impairment in the follow-up detection of local cancer recurrence $(6,7)$. This precedent remained until 2007 when Coleman and Saboeiro published a report showing safety and satisfactory outcome of AFG in the setting of breast oncoplastic reconstruction using a Coleman's method with long-term follow-up data in a group of 17 patients (8). Given the cosmetic benefits and normal postoperative mammogram findings demonstrated in this study, the ASPS revised their previous stance in cautious support of AFG in breast reconstruction and oncoplastic augmentation (9).

Following the change of the ASPS guidelines, many studies have continued to investigate the safety and efficacy of AFG for breast reconstruction following oncological resections with conflicting results. Cohen et al. compared the outcomes of patients who underwent mastectomy with or without AFG and found a similar rate of local recurrence compared to patients who did not undergo AFG. However, in the AFG group, recurrence was diagnosed at a significantly later time compared to the group that did not undergo AFG (10). A concerning explanation for this finding may be that AFG interferes with early detection of recurrence and interferes with the accuracy of diagnostic modalities (11). While the authors of this study argued that if this was true, then the cancer would have been diagnosed at a more advanced stage (which it was not), lengthier long-term follow-up was required to validate such assumptions. However, a study looking specifically at the interpretation of mammograms following AFG and reduction mammaplasty by academic radiologists found that

^ ORCID: Alireza Hamidian Jahromi, 0000-0002-7585-847X; Sydney R. Horen, 0000-0001-6996-6039. 
more scarring, masses requiring biopsy, and higher BIRADS scores were found for the reduction mammaplasty images compared to those in which AFG had been performed (12). Furthermore, other studies $(13,14)$ have supported the safety of AFG over longer follow-up than the previously mentioned study by Cohen et al. Krastev et al. performed a systematic review looking at the rates of cancer recurrence in patients who underwent AFG following mastectomy or BCS and found that these were comparable to control groups (13). Similarly, a multicenter study of 488 patients who underwent AFG for breast reconstruction reported a low local recurrence rate of $0.5 \%$ over an averaged 7 -year follow-up (14). This exceedingly low rate of malignancy recurrence with similar rates seen in control groups supports the notion that at this time the evidence shows that AFG does not add an additional risk of cancer recurrence in the setting of oncologic reconstruction.

Complications related to AFG as well as fat volume retention must be considered in patients undergoing this procedure. While malignancy reoccurrence may be the most noteworthy complication, less devastating events still associated with an increased morbidity may occur. These include oil cyst development, infection, fat necrosis, wound healing problems, seroma, or hematoma formation (10). Fat volume loss may also occur. In addition to patient demographics and comorbidity profiles, the occurrence of these negative outcomes is largely related to surgical technique. While Coleman's principles revolutionized the technique of $\mathrm{AFG}$, inconsistencies amongst surgical practices remain (15). The lack of consensus for a single method of fat harvest, processing, and grafting, can lead to variable results. Systematic review of the efficacy of AFG to the breast has demonstrated an overall fat retention rate of $76.8 \%$, however, this mean includes a range of studies reporting retention as high as $82.6 \%$ to as low as $44.7 \%$ (16). Lower rates of $\mathrm{AFG}$ retention may result in the need for more AFG sessions (in this systematic review, AFG sessions ranged from 1 to 6 ) which can increase morbidity and decreased patient satisfaction (16). In addition, when comparing studies using AFG to the breast for cosmetic purposes rather than oncologic reconstruction, AFG is less effective in the oncoplastic settings (1). This variation may be even greater when factoring in variables such as radiation, which may significantly decrease volume retention (17). Due to the heterogeneity of AFG outcome reporting (18) in conjunction with technique variations, it is difficult to compare results across studies. However, the fact remains that fat retention rates are highly variable.
For patients who undergo mastectomy, AFG used in different capacities for breast reconstruction may yield different results. Options include total reconstruction, augmentation to implants, augmentation to flaps, or, should complications arise, simultaneous implant exchange with fat (5). Total reconstruction with AFG is not routinely performed; however, promising results have been shown using approximately 3-4 sessions to achieve a mean volume of $225 \mathrm{cc}$ per breast or with the implementation of the BRAVA system (14). A notable concern with these "megavolume" AFG procedures has been fat necrosis which has been reported to be as high as $33 \%$ in patients with a grafted volume of $337 \mathrm{cc}$ or higher (19). This amount of fat necrosis was shown to increase as the amount of grafted fat increased and was even more pronounced in patients who had undergone radiation. Large volume AFG is much more commonly employed to augment implant or flapbased reconstruction. For patients undergoing implant reconstruction, AFG can be implemented at the time of permanent implant placement, or at a later stage to add volume to contour deformities or thicken thin mastectomy flaps. Similarly, in flap-based reconstructions, AFG may be used to create a seamless transition between the native chest wall and the transferred tissue flap. Simultaneous implant exchange with AFG may be used in cases in which complications such as tissue thinning, capsular contracture and asymmetry/malposition arise. This method has been used in place of traditionally used solutions (i.e., implant exchange or change in plane, conversion to flap reconstruction, or complete removal, etc.) with good results (20).

Given the equivalent oncological outcomes of mastectomy and BCS with radiation, the use of AFG in the BCS patient group has become increasingly popular. Like patients who undergo mastectomy, AFG may be employed secondarily to fill defects caused by radiation or prior lumpectomy (partial mastectomy) (21). Immediate AFG in BCS is another technique that has shown promising results in terms of cancer recurrence rates, complications, and patient satisfaction (22-24). The recently published study by $\mathrm{Li}$ et al., adds to the growing literature in support of immediate AFG in BCS (25). In this study the authors report on their experience with this technique in what is the first published Asian patient cohort to undergo immediate AFG following BCS. As mentioned by the authors, Asian women may be an ideal group for this reconstructive option as they generally have smaller breasts than western women. While western women may require larger amounts of fat to be grafted, multiple fat grafting sessions, and potentially 
necessitate flap or implants-based reconstruction, the smaller body habitus of Asian women may benefit from the lower-morbidity and simplicity associated with onestage immediate AFG. Also, the smaller size of breasts of Asian women makes the aesthetic outcome following a same size partial mastectomy less satisfactory as deficiencies are more difficult to conceal. Therefore, these patients may benefit from a reconstructive intervention (i.e., a larger proportion would benefit from $\mathrm{AFG}$ ). While it is surprising that significantly fewer women in China choose to undergo BCS for early-stage breast cancer compared to women in America, as the authors suggest, it seems likely that this number will rise in the future, especially with the option of undergoing AFG to improve breast shape and symmetry.

The cohort of patients in the study by Li et al. compared 30 women who underwent immediate AFG following BCS to 28 women who underwent BCS alone. The small size of these patient groups and the retrospective and nonrandomized nature of the study make generalization of these results unreliable. However, the authors made a commendable effort to match their experimental and control cohorts regarding patient and surgical characteristics. This is especially important with regard to tumor pathology and stage, both of which can directly impact malignancy recurrence rate. Of note, the patients included in this study were relatively young with the average age in the AFG+BCS and the BCS groups being 44.6 and 45.3, respectively. The patients were also of a low BMI subset, with the average BMIs being 22.9 and $22.6 \mathrm{~kg} / \mathrm{m}^{2}$ in the $\mathrm{AFG}+\mathrm{BCS}$ and the BCS groups, respectively. In the previous studies of this technique performed in Europe and South America, patient groups have been of a higher BMI characteristic $(22,24)$. Similarly, women of other western countries such as the United States on average have a higher BMI than that of Chinese women. These desirable patient demographics make comparison to most other countries across the world in which women would generally be of older age and higher BMI less applicable.

The results reported by $\mathrm{Li}$ et al. showed only one complication in the AFG+BCS group (seroma), no donor site complications, and one instance of recurrence in the AFG + BCS and BCS groups that was regional and systemic, respectively. These results support the safety of this technique in terms of complication profile and risk of malignancy recurrence. These topics remain of important consideration with unclear guidelines regarding true rates and risk profiles. While results in other studies that have used AFG in other capacities (i.e., following mastectomy with flap or implant-based reconstruction) have shown similarly optimistic outcomes, further study with larger patient cohorts and prospective data collection for the technique of $\mathrm{AFG}+\mathrm{BCS}$ is required to validate safety. In addition, the follow-up period used in this study, which ranged from 36 to 44 months, may have missed any longerterm recurrences being diagnosed. In the previously mentioned study by Cohen et al., malignancy recurrence was diagnosed on average at 52.3 months in patients who underwent AFG compared to 22.8 months in those who did not (10). As the present study only conducted follow-up through 44 months, if their cohort followed a similar trend, some cases of malignancy may have been missed. Even with the follow-up provided by Cohen $e t$ al., the possibility of a second recurrence peak at 60 months makes even lengthier follow-up for both of these studies necessary for a complete evaluation of the oncologic risks of AFG (11).

It is also worth mentioning that $\mathrm{Li}$ et al. employed strict inclusion and exclusion criteria including only evaluating patients with stage I/II cancer, a single tumor which was less than $4 \mathrm{~cm}$ in diameter, more than $3 \mathrm{~cm}$ from the nipple, and those without metastasis, systemic disease, other cancers, or additional contraindications. Naturally, some of these criteria must be implemented for the feasibility of BCS and for breast reconstruction in general, however, it should be recognized that the patients in this study were excellent candidates from an oncologic standpoint, with a very low risk of recurrence already, without consideration of additional procedures like AFG.

The results reported by $\mathrm{Li}$ et al. with regard to physician satisfaction show excellent aesthetic outcomes. These corroborate previously reported outcomes showing significantly greater cosmetic satisfaction across all domains in patients who undergo BCS + AFG compared to those who undergo BCS alone $(22,23)$. While these results may be somewhat expected, the greater than two-fold increase in physician satisfaction regarding cosmetic outcomes in the group that received AFG compared to the group that did not $(33 \%$ versus $15 \%)$ speaks to the positive impact of this technique.

With regard to patient reported-outcomes, the BREAST-Q was employed and showed significant outcomes in only three out of six reported questions aimed to evaluate satisfaction with physical appearance. Aside from the inherent flaws with this survey, these results may be explained by the fact that the remaining defects that some patients who underwent BCS had were minimal and did not have a large impact on the overall satisfaction. Additionally, the authors 
briefly mention the impact of radiation, stating that radiation did interfere with aesthetic results for most AFG cases. Further qualification of the impact of radiation in this group should be included, especially given the known repercussions previously reported in other studies evaluating radiation in conjunction with AFG (17). Finally, AFG efficacy may explain some of the lower rates of patient reported satisfaction in the $\mathrm{AFG}+\mathrm{BCS}$ group. If a high percentage of fat tissue did not survive due to radiation or grafting technique, patients in the AFG + BCS group may have been less satisfied with their outcomes. Longitudinal quantification and qualification of these variables over extended follow-up periods may further elucidate potential trends.

Li et al. reported significantly higher psychosocial wellbeing in patients who underwent $\mathrm{AFG}+\mathrm{BCS}$ compared to those who underwent BCS alone. As breast reconstruction in general has been linked to higher psychosocial wellbeing and patient satisfaction, it is unsurprising that the technique of BCS + AFG would have similar results. Especially given the young, relatively healthy, and low risk patient population, patients who did not undergo any reconstruction would expectedly be less satisfied with their outcomes following BCS. In addition, the single-stage reconstruction that BCS + AFG offers would seemingly offer higher patient satisfaction due to less interference with daily life and financial expenditure on the patient's part.

Overall, the results reported in the study by $\mathrm{Li}$ et al. assessing the use of immediate AFG in BCS support the positive outcomes that this technique may have for the properly selected patient. The authors acknowledge the limitations to their study and their assessment of its flaws is accurate. Cautious application of this technique should be encouraged due to the unique patient demographics, short follow-up, and the non-randomized and retrospective design; however, ongoing research from this team should soon address the latter considerations. While the study presents optimistic results, the continued consideration of oncologic safety, complication profile, and fat graft efficacy should remain a high priority for all those who choose to implement these and other AFG techniques.

\section{Acknowledgments}

Funding: None.

\section{Footnote}

Provenance and Peer Review: This article was commissioned by the editorial office, Gland Surgery. The article did not undergo external peer review.

Conflicts of Interest: Both authors have completed the ICMJE uniform disclosure form (available at https://dx.doi. org/10.21037/gs-21-563). The authors have no conflicts of interest to declare.

Etbical Statement: The authors are accountable for all aspects of the work in ensuring that questions related to the accuracy or integrity of any part of the work are appropriately investigated and resolved.

Open Access Statement: This is an Open Access article distributed in accordance with the Creative Commons Attribution-NonCommercial-NoDerivs 4.0 International License (CC BY-NC-ND 4.0), which permits the noncommercial replication and distribution of the article with the strict proviso that no changes or edits are made and the original work is properly cited (including links to both the formal publication through the relevant DOI and the license). See: https://creativecommons.org/licenses/by-nc-nd/4.0/.

\section{References}

1. Moak TN, Ebersole TG, Tandon D, et al. Assessing Clinical Outcomes in Autologous Fat Grafting: A Current Literature Review. Aesthet Surg J 2021;41:S50-60.

2. Anderson C, Hamidian Jahromi A, Miller EJ, et al. The Current Status of the Autologous Fat Grafting for Pediatric Craniofacial Patients. Ann Plast Surg 2020;85:568-73.

3. Gornitsky J, Viezel-Mathieu A, Alnaif N, et al. A systematic review of the effectiveness and complications of fat grafting in the facial region. JPRAS Open 2018;19:87-97.

4. Toledo LS. Gluteal augmentation with fat grafting: the Brazilian buttock technique: 30 years' experience. Clin Plast Surg 2015;42:253-61.

5. Katzel EB, Bucky LP. Fat Grafting to the Breast: Clinical Applications and Outcomes for Reconstructive Surgery. Plast Reconstr Surg 2017;140:69S-76S.

6. Illouz YG. Body contouring by lipolysis: a 5-year experience with over 3000 cases. Plast Reconstr Surg 1983;72:591-7.

7. Report on autologous fat transplantation. ASPRS Ad-Hoc Committee on New Procedures, September 30, 1987. Plast Surg Nurs 1987;7:140-1. 
8. Coleman SR, Saboeiro AP. Fat grafting to the breast revisited: safety and efficacy. Plast Reconstr Surg 2007;119:775-85; discussion 786-7.

9. Gutowski KA; ASPS Fat Graft Task Force. Current applications and safety of autologous fat grafts: a report of the ASPS fat graft task force. Plast Reconstr Surg 2009;124:272-80.

10. Cohen O, Lam G, Karp N, et al. Determining the Oncologic Safety of Autologous Fat Grafting as a Reconstructive Modality: An Institutional Review of Breast Cancer Recurrence Rates and Surgical Outcomes. Plast Reconstr Surg 2017;140:382e-92e.

11. Hamidian Jahromi A. Determining the Oncologic Safety of Autologous Fat Grafting as a Reconstructive Modality: An Institutional Review of Breast Cancer Recurrence Rates and Surgical Outcomes. Plast Reconstr Surg 2018;142:579e-80e.

12. Rubin JP, Coon D, Zuley M, et al. Mammographic changes after fat transfer to the breast compared with changes after breast reduction: a blinded study. Plast Reconstr Surg 2012;129:1029-38.

13. Krastev TK, Jonasse Y, Kon M. Oncological safety of autologous lipoaspirate grafting in breast cancer patients: a systematic review. Ann Surg Oncol 2013;20:111-9.

14. Khouri RK, Rigotti G, Khouri RKJr, et al. Tissueengineered breast reconstruction with Brava-assisted fat grafting: a 7-year, 488-patient, multicenter experience. Plast Reconstr Surg 2015;135:643-58.

15. Denadai R. Pursuing the Optimal Fat Grafting Technique for Cleft-Craniofacial Reconstruction: Toward a Physiologically and Anatomically Driven Surgical Approach. Ann Plast Surg 2021;86:121.

16. Groen JW, Negenborn VL, Twisk DJWR, et al. Autologous fat grafting in onco-plastic breast reconstruction: A systematic review on oncological and radiological safety, complications, volume retention and patient/surgeon satisfaction. J Plast Reconstr Aesthet Surg
2016;69:742-64.

17. Garza RM, Paik KJ, Chung MT, et al. Studies in fat grafting: Part III. Fat grafting irradiated tissue--improved skin quality and decreased fat graft retention. Plast Reconstr Surg 2014;134:249-57.

18. Agha RA, Pidgeon TE, Borrelli MR, et al. Validated Outcomes in the Grafting of Autologous Fat to the Breast: The VOGUE Study. Development of a Core Outcome Set for Research and Audit. Plast Reconstr Surg 2018;141:633e-8e.

19. Chang CS, Lanni MA, Lamelas AM, et al. Abstract: Identifying Risk Factors for Fat Necrosis After Autologous Fat Transplantation to the Breast. Plast Reconstr Surg Glob Open 2016;4:135-6.

20. Del Vecchio DA. "SIEF"--simultaneous implant exchange with fat: a new option in revision breast implant surgery. Plast Reconstr Surg 2012;130:1187-96.

21. Hanson SE, Kapur SK, Garvey PB, et al. Oncologic Safety and Surveillance of Autologous Fat Grafting following Breast Conservation Therapy. Plast Reconstr Surg 2020;146:215-25.

22. Biasio F, Bertozzi S, Londero AP, et al. Surgical and oncological outcomes of free dermal fat graft for breast reconstruction after breast-conserving surgery. Adv Clin Exp Med 2018;27:773-80.

23. Khan LR, Raine CR, Dixon JM. Immediate lipofilling in breast conserving surgery. Eur J Surg Oncol 2017;43:1402-8.

24. Biazus JV, Stumpf CC, Melo MP, et al. Breast-Conserving Surgery with Immediate Autologous Fat Grafting Reconstruction: Oncologic Outcomes. Aesthetic Plast Surg 2018;42:1195-201.

25. Li H, Wang Y, Yang D, et al. Clinical study of the feasibility, complications, and cosmetic outcomes of immediate autologous fat grafting during breastconserving surgery for early-stage breast cancer in China. Gland Surg 2021;10:2386-97.
Cite this article as: Hamidian Jahromi A, Horen SR. Editorial: feasibility, complications, and cosmetic outcomes of immediate autologous fat grafting during breast-conserving surgery for early-stage breast cancer. Gland Surg 2021;10(10):2885-2889. doi: $10.21037 / g s-21-563$ 